\title{
Outcome prediction following penetrating craniocerebral injury in a civilian population: aggressive surgical management in patients with admission Glasgow Coma Scale scores of 6 to 15
}

\author{
MiCHAEL L. LEVY, M.D. \\ Department of Neurological Surgery, University of Southern California School of Medicine, \\ Los Angeles California
}

\begin{abstract}
In an attempt to assess admission Glasgow Coma Scale (GCS) scores and other radiographic variables after penetrating craniocerebral injury in relationship to outcome, the author evaluated a series of 294 patients with penetrating injuries who presented with a GCS score of 6 to 15 over a 6-year period. Entrance criteria required a replicable neurological examination that was not altered by the presence of hypotension, drugs/toxins, or systemic injury. All patients underwent surgical intervention and aggressive perioperative management, including resuscitative protocols, in the neurosurgical intensive care unit.

The author previously devised prospective models of outcome remained unchanged in this series. The variables most predictive of death include admission GCS score and subarachnoid hemorrhage in one model and admission GCS score and pupillary changes in a second when pupillary response was definitive at admission $(\mathrm{p} \leq 0.00005)$. Other important variables related to morbidity include admission GCS, bihemispheric injury when associated with intraventricular hemorrhage, and diffuse fragmentation $(\mathrm{p} \leq 0.001)$.

In this study a significant relationship between operative intervention and survival $(p \leq 0.01)$ was found in patients with an admission GCS scores of 6 to 8. No significant relationships between operative intervention and survival were found in patients with admission GCS scores of 9 to 12 and 13 to 15 . A significant relationship between operative intervention and morbidity $(\mathrm{p} \leq 0.01)$ was also demonstrated in patients with an admission GCS score of 12 to 15 . No significant relationships between operative intervention and morbidity were found in patients with an admission GCS score of 6 to 8 and 9 to 12 .
\end{abstract}

\section{KEY WORDS • head injury • penetrating craniocerebral injury • Glasgow Coma Scale • outcome}

Patients with craniocerebral missile wounds who arrive at the hospital alive often receive variable treatment despite low GCS scores and often dismal prognoses, because there is a lack of consensus regarding appropriate treatment and predictable outcome in these patients. We have previously reviewed a series of patients with admission GCS scores of 3 to 5 and reported that although surgery was significantly related to mortality, it had no effect on morbidity, with poor outcome being demonstrated in the vast majority of surgically treated survivors. ${ }^{12}$ In the current paper we discuss outcome following aggressive surgical management in patients whose GCS scores were 6 to 15 .

Abbreviations used in this paper: $\mathrm{CT}=$ computerized tomography; GCS = Glasgow Coma Scale; GOS = Glasgow Outcome Scale; $\mathrm{SAH}=$ subarachnoid hemorrhage.

\section{CLINICAL MATERIAL AND METHODS}

Between 1985 and 1992 there were 1086 penetrating head trauma patients who presented to the University of Southern California-Los Angeles County Medical Center. Included in the series were all patients with craniocerebral missile wounds Of the 1086 patients only those with inclusion of all analyzed variables and a three-year follow up were included in the analysis. This yielded a series of 484 patients. Of this series, 343 patient records were reviewed retrospectively and 141 patients were entered prospectively. Of these, 190 with admission GCS scores of 3-5 have been previously discussed in an earlier paper. The remaining 294 patients with admission GCS of 6-15 are now evaluated as the purpose of this study. The utilization of models in predicting the outcome of patients entered on a prospective basis had no influence on their medical or surgical management. The role of selection 
bias should be noted given that this study was performed at a single institution where methodologies for treating these patients has been well established.

All patients included in the study were given a replicable neurological examination following medical resuscitation; the examination was unencumbered by drugs, toxins, or systemic injury. Patients with intractable hypotension in whom resuscitation with fluids or vasopressive agents failed were not included in the study.

Criteria for inclusion in the series included isolated penetrating craniocerebral injury and neurological exams that were not compromised by hypotension or the presence of drugs or toxins. Computerized tomography scans were performed on all patients. Outcome determination was based on full neurological evaluations according to the GOS score at the time of admission, discharge, and at follow up of up to 3 years.

\section{Acute Management}

In the present study, if a patient's pupils were reactive to light, surgery was performed within 24 hours, whenever possible. Surgery was also performed in some patients with unreactive pupils as well, but only if optic nerve injury was suspected or if there were other mitigating factors, such as intracranial bleeding or toxin-induced alterations in mental status. In a survey by Kaufman, et al. ${ }^{8}$ the authors found that $23 \%$ of neurosurgeons surgically intervene when both pupils are dilated, whereas $72 \%$ will perform surgery in patients in whom one pupil is dilated.

Surgical intervention was kept to a minimum, and it primarily involved debridement at the superficial wound site and evacuation of mass lesions . Fragments of bone or bullet lodged deep in the parenchyma or in a site not readily accessible were left in place. After surgery, patients were aggressively managed in the neurosurgical intensive care unit. ${ }^{12}$

\section{Statistical Analysis}

A total of 38 variables were recorded in this series. All variables were screened for outliers by using Grubbs analysis. Continuous variables were evaluated for normal distribution. The variable of age was normalized using a logarithmic transformation. Forward and backward linear stepwise regression analysis (BMDP 2R; BMDP Statistical Software, Inc., Los Angeles, CA) was used to relate all independent variables to outcome (GOS score). Twogroup tests and matched t-tests were used to correlate all independent variables with outcome in addition to individual variables present within a subgroup. The equality of group variances was evaluated by using Levene's test (BMDP 3D). Levels of statistical significance were set at $\mathrm{p}<0.05$. Adequacy of outcome was expressed in terms of the GOS scores and was described as either good (GOS score of 4-5) or poor (GOS score of 1-3). The GOS score was assigned within 12 months following discharge from the hospital. A good outcome indicated that the patient was either without neurological sequelae or had a moderate residual deficit but was independent. To identify the most significant survival indicators, multivariate stepwise logistic regression analysis was conducted.

On the basis of our clinical experience we were able to classify distinct sets of variables into subgroups. Sub- groupings included variables that contributed to increased intracranial pressure; variables that lead to diffuse intracerebral fragmentation; entry sites with involvement of both hemispheres; and injuries affecting multiple lobes with ventricular involvement.

\section{RESULTS}

The overall series was comprised of $89 \%$ male and $11 \%$ female patients. Mean age was 26 years (range 2-97 years). Mean transit time between the scene of injury and hospitalization was 25 minutes (range 0-92 minutes). Mean length of hospital stay was 7 days (range 1-179 days). Injuries occurred between 3 a.m. and 6 p.m. (32\%) or 6 p.m. and 3 a.m. (68\%). Motivation for the shootings was gang related in $52 \%$. Non gang-related injuries were sustained during assault $(21 \%)$, suicide $(19 \%)$, robbery $(2 \%)$, accident $(3 \%)$, drug-related encounters $(1 \%)$, police-related encounters $(1 \%)$, and those occurring during random activities $(1 \%)$. Missle wound entrance sites included frontal (34\%), temporal (23\%), parietal (19\%), occipital (20\%), and other (4\%). Patient race included Hispanic (78\%), African American (14\%), Caucasian (3\%), and Asian (5\%).

Fifty-three patients were admitted with an admission GCS score of 6 to 8,64 with a score of 9 to 12 , and 177 with a score of 13 to 15 . Two hundred eight patients underwent operative intervention dural closure was obtained in all patients whereas 86 were managed nonsurgically. Patients undergoing craniotomy/craniectomy surgery included 48 patients with an admission GCS score of 6 to 8, 54 with an admission GCS score of 9 to12, and 106 with an admission GCS score of 13 to 15 . With regard to mortality in cases of operative management, 40 patients (83\%) with GCS scores of 6 to 8 survived, $45(83 \%)$ with GCS scores of 9 to 12 survived, and 102 (96\%) with GCS scores of 13 to 15 survived (Table 1). As compared with our earlier study ${ }^{11}$ this represents an increase of 15 patients who underwent surgery (29 lived and six died) and of four who did not undergo surgical intervention (all died) in the patients with admission GCS scores of 6 to 8).

With regard to morbidity in patients who underwent operative intervention, 12 patients (25\%) with GCS scores of 6 to 8 experienced a good outcome, $25(46 \%)$ with GCS scores of 9 to 12 experienced a good outcome, and 85 (81\%) with GCS scores of 13 to 15 experienced a good outcome. The only significant relationship between operative intervention and outcome was found in patients with an admission GCS scores of 13 to 15 ( $p \leq 0.05$ ).

With regard to the adequacy of outcome as determined using the GOS in the entire series, there was good outcome in 12 patients (23\%) with admission GCS scores of 6 to 8 , good outcome in 30 (47\%) with admission GCS scores of 9 to 11 , and good outcome in $152(86 \%)$ with admission GCS scores of 12 to 15 (Table 2). Overall, 40 patients $(75 \%)$ with an admission GCS scores 6 to 8 survived, $52(81 \%)$ with a GCS scores of 9 to 12 survived, and $172(97 \%)$ with a GCS scores of 13 to $15(97 \%)$ survived. Outcome with regard to the duration of hospitalization was notable because patients with a GOS score of 1 were hospitalized for a mean duration of 2 days (range 1-33 days), patients with a GOS score of 2 were hospitalized for a mean of 85 days (range 12-258 days), patients 
TABLE 1

INCIDENCE OF MORTALITY AFTER SURGICAL AND NONSURGICAL INTERVENTION

\begin{tabular}{cccr}
\hline \hline \multirow{2}{*}{$\begin{array}{c}\text { Admission } \\
\text { GCS Score }\end{array}$} & Survived & Died & p value \\
\cline { 2 - 4 } surgical intervention & & & \\
$6-8$ & $40(83)$ & $8(17)$ & $<0.01$ \\
$9-11$ & $45(83)$ & $9(17)$ & $\mathrm{NS}$ \\
$12-15$ & $102(96)$ & $4(4)$ & $\mathrm{NS}$ \\
nonsurgical intervention & & & \\
$6-8$ & $0(0)$ & $5(100)$ & $<0.01$ \\
$9-11$ & $7(71)$ & $3(29)$ & $\mathrm{NS}$ \\
$12-15$ & $70(98)$ & $1(2)$ & $\mathrm{NS}$ \\
\hline
\end{tabular}

* NS = not significant.

with a GOS score of 3 were hospitalized for a mean of 29 days (range 3-129 days), patients with a GOS score of 4 were hospitalized for a mean of 13 days (range 1-51 days) and patients with a GOS score of 5 were hospitalized for a mean of 5 days (range 1-54 days).

\section{Outcome Prediction}

We found that the variables most predictive of death as determined by logistic regression analysis included 1) admission GCS score and SAH ( $\mathrm{p} \leq 0.00005)$ when pupillary function was not intact and 2) admission GCS score and pupillary changes $(\mathrm{p} \leq 0.00005)$ when pupillary function was intact. The variables most predictive of morbidity, as determined by logistic regression analysis included admission GCS score, bihemispheric injury associated with intraventricular hemorrhage, $\mathrm{SAH}$, and diffuse fragmentation $(\mathrm{p}<0.001)$.

\section{DISCUSSION}

In most series the investigators have shown that patients fit into two groups: those presenting with GCS scores of 12 to15 and those who do not survive..$^{19}$ Thus, there are only a few patients in the population with GCS scores of 5 to 12 whose outcomes may be helped by appropriate neurosurgical efforts. In a series from the University of
Virginia, only one patient died after the 1st week following injury; this patient had a GCS score of 15 and died of a pulmonary embolism. ${ }^{19}$ Among the survivors in this series, none was vegetative and only two were severely disabled. The remainder made a good outcome (mild or no disability). ${ }^{21}$ Selden, et al., ${ }^{20}$ also reported a good outcome in those patients who survived a suicide attempt, with $91 \%$ (10 of 11) experiencing a good outcome. In the series reported by Kaufman, et al., ${ }^{7}$ only $5 \%$ of 48 survivors were vegetative or severely disabled, $29 \%$ experienced a moderate disability, and $67 \%$ experienced a good outcome. Only 38\% remained in the hospital for either rehabilitation or lengthy acute care treatment .

I have devised prospective models of outcome based on an attempt to predict on admission patients who will be nonsurvivors. To decrease the chance that any future models would indicate that a poor outcome, based on the variables present on admission in a patient who in turn may make a good outcome, I established the significance of the model at $\mathrm{p}<0.001$, with a confidence interval of greater than $99 \%$ (thus overpredicting for survivors). My aim was to create a formula with which good outcome will be predicted (that is, to know who will survive what percentage of the time and who will die what percentage of the time). Care is considered to be futile when there is a less than $1 \%$ chance for survival. By description, such models are used to predict outcome while controlling for confounding in-

TABLE 2

INCIDENCE OF MORBIDITY AND MORTALITY AFTER SURGICAL AND NONSURGICAL INTERVENTION

\begin{tabular}{cccc}
\hline \hline & \multicolumn{3}{c}{ Outcome (\%) } \\
\cline { 2 - 4 } $\begin{array}{c}\text { Admission } \\
\text { GCS Score }\end{array}$ & $\begin{array}{c}\text { Good } \\
\text { (GOS 4 or 5) }\end{array}$ & $\begin{array}{c}\text { Poor } \\
\text { (GOS 2 or 3) }\end{array}$ & Died \\
\hline surgical intervention & & & \\
$6-8$ & $12(24)$ & $28(57)$ & $8(17)$ \\
$9-11$ & $25(46)$ & $20(37)$ & $9(17)$ \\
$12-15$ & $85(80)$ & $17(16)$ & $4(4)$ \\
nonsurgical intervention & & & \\
$6-8$ & $0(0)$ & $0(0)$ & $5(100)$ \\
$9-11$ & $5(50)$ & $2(20)$ & $3(30)$ \\
$12-15$ & $67(95)$ & $3(4)$ & $1(1)$ \\
\hline
\end{tabular}


teractions between variables that are considered for inclusion into the model.

\section{Glasgow Coma Scale}

The admission GCS score remains the strongest and most significant predictor of outcome in the author's work to date. The results of numerous studies have also supported the findings that patients with admission GCS scores of 3,4 , or 5 experience poor outcomes. ${ }^{1,4,6,7,10,12,14,18,22}$ I have previously attempted to answer this question in patients with admission GCS scores of 3 to 5 who underwent aggressive management (both surgical and nonsurgical). ${ }^{12}$ In this earlier series there were 60 operative patients, 21 with an admission GCS score of 3, 24 with a score of 4, and 15 with a score of 5 . None of the five patients with GCS scores of 3 who survived had good outcomes (GOS score of 4 or 5). The patients with GCS scores of 4 fared only slightly better, with one of the seven surviving patients making a good outcome. One of the 11 survivors in the group with GCS scores of 5 experienced a good outcome. Although operative intervention saved lives when compared with nonoperative treatment, 23 patients $(62 \%)$ versus one patient $(<1 \%)$ survived, and only two survivors made a reasonable neurological recovery. Moreover, although these two patients could function independently, they remained severely compromised.

In a retrospective study of 62 patients between the ages of 10 and 72 years the authors reported that $32 \%$ of patients died in the ambulance or upon arriving at the medical center. ${ }^{21}$ Eighty-eight percent of patients (14 of 16) with an admission GCS score of 3 or 4 died within 1 week of injury. ${ }^{21}$ Stone, et al., ${ }^{24}$ also evaluated a series of patients with craniocerebral missile wounds who underwent aggressive surgical intervention. The overall mortality rate was $34 \%$, with a $21 \%$ mortality rate for the 150 surgical patients. Of patients undergoing surgery, three $(50 \%)$ of six patients with admission GCS scores of three survived, and $16(42 \%)$ of 38 patients with admission GCS scores of 4 to 7 survived. In operative patients presenting with a GCS score of 8 to 14 there was a $90 \%$ survival rate, whereas all of the patients with a GCS score of 15 survived. When comparing GCS scores before and after initial resuscitation, the authors concluded that outcome was strongly associated with increases or decreases in GCS score. ${ }^{24}$ As I have also found, the postresuscitation GCS score was the strongest predictor of outcome. Only one $(1.5 \%)$ of 66 patients presenting in traumatic arrest was resuscitated and had a GOS score of 3. Patients experiencing hypotension with a systolic blood pressure of less than $90 \mathrm{~mm} \mathrm{Hg}$ also fared poorly, with injury-related deaths at admission or during the early stages of their acute hospitalization occurring in $62 \%$. Furthermore, outcome in those patients with hypotension who survived was not as good as in those patients with normal or high blood pressure $(\mathrm{p}<0.005)$.

\section{Subarachnoid Hemorrhage}

We have also assessed the predictive value of SAH in outcome for victims of intracranial missile injury in the present study and prior studies. ${ }^{13}$ Previously, in a study of 100 patients who had sustained craniocerebral missile wounds, the overall mortality rates were $28 \%$ after exclud- ing patients who died on or immediately after admission. Of the patients who died within 48 hours of admission, in $68 \%$ (19 of 28) SAH was demonstrated on admission, as compared with $17 \%$ (12 of 72) of the patients who survived. Thus, there was a statistically significant correlation between SAH and both mortality and morbidity. Vasospasm, as determined angiographically, was noted in $16 \%$ of patients (five of 31) with SAH. ${ }^{13}$

Although multiple variables have been reported to be associated with poor outcome, SAH was correlated with poor outcome even in the absence of these other variables. On the contrary, Stone, et al. ${ }^{24}$ have reported that among operative patients, the presence of SAH, subdural hematoma, or epidural hematoma on CT was not a significant predictor of outcome.

\section{Prediction of Vasospasm}

Cerebral arterial spasm is a potentially devastating complication frequently associated with SAH..$^{15-17,25}$ The association of SAH with craniocerebral missile wounds has been investigated using transcranial Doppler ultrasonography to measure blood velocity in the middle cerebral artery and in the extracranial internal carotid artery in a series of 33 patients. ${ }^{9}$ The frequency of vasospasm was shown to be higher after penetrating injury $(42.4 \%)$ than after closed head trauma $(27 \%)^{9,17,2]}$ or aneurysmal SAH $(36 \%) .{ }^{9,26}$ In all 14 patients suffering vasospasm evidence of SAH was demonstrated on CT scanning. It is important to note that the admission GCS score was not a good predictor of the occurrence of cerebral arterial vasospasm in this series. Fifty-six percent of patients without evidence of vasospasm made a favorable outcome, whereas only $36 \%$ of patients with evidence of vasospasm made a favorable outcome (GOS score of 4 or 5).

\section{Other Variables Related to Outcome}

Pupillary function is a strong predictor of outcome. I have evaluated models of outcome both in the absence and presence of pupillary response given its significance to outcome. Polin and colleagues ${ }^{19,21}$ have reported that only $21 \%$ of those patients who presented with bilaterally fixed and dilated pupils survived. Patients presenting with unilateral pupillary dysfunction had a $50 \%$ survival rate, as compared with a $95 \%$ survival rate in those presenting with reactive pupils. In the series of 480 patients reported by Stone, et al. ${ }^{24}$ of four patients (4\%) with bilaterally unreactive pupils who survived their initial injuries, three were vegetative (GOS score of 2). Patients with dilated, nonreactive, or unequal pupils had relatively poor outcomes in this series. ${ }^{24}$

Coagulopathy is also a significant predictor of outcome. ${ }^{9,21}$ In one study the investigators documented that abnormal activated partial thromboplastin time or prothrombin time on Day 1 was associated with only a $20 \%$ survival rate. ${ }^{19}$ Kaufman, et al., ${ }^{7}$ have reported that the incidence of an abnormality in coagulation status was significantly higher in nonsurvivors than in survivors $(94 \%$ compared with $71 \%$, respectively). One must use stringent criteria to determine if disseminated intravascular coagulation given, in our experience, that the presence of disseminated intravascular coagulation has an associated mortality rate of greater than $85 \%$. 
In an other series the authors have reported significant correlations between ventricular penetration and death, with death as the outcome in $84 \%$ of patients with ventricular injury. ${ }^{19}$ In different series of 76 patients it was reported that $90 \%$ of patients with ventricular involvement made a poor outcome. ${ }^{3}$ Nagib, et al. ${ }^{18}$ have reported good outcomes in only one of 14 patients with ventricular involvement. In the present series, ventricular involvement was found to be a significant predictor of outcome only when coupled with bihemispheric injury.

Bihemispheric involvement has been found to be predictive of poor outcome in numerous series. ${ }^{5,7,18,21,23}$ In the series reported by Clark, et al., ${ }^{3} 86 \%$ of the patients with bihemispheric injury experienced poor outcomes as compared with 36\% with unilateral involvement in whom poor outcomes were demonstrated. Kaufman, et al., ${ }^{7}$ have found only a $15 \%$ survival rate in patients in whom both hemispheres were involved, whereas $64 \%$ of patients with unilateral involvement survived. Similar results have been reported in other studies, with a less than $2 \%$ survival rate when the midline was crossed; only $15 \%$ of patients survived a unilateral injury. ${ }^{2}$ Polin, et al., ${ }^{19}$ have found a higher incidence of death if either the axial or midsagittal plane was crossed, with bisection of both planes resulting in a higher mortality rate. Other authors have reported that in $57 \%$ of surgical patients (four of seven) with bihemispheric involvement a good outcome was demonstrated. ${ }^{5}$

Analysis of results in the present series suggests that patients with craniocerebral missile wounds and a low GCS score (3 to 8) are not a homogenous group. Eighty-three percent of patients undergoing surgery with admission GCS scores of 6 to 8 survived. Twenty-four percent made good outcomes whereas 57\% made poor outcomes and $17 \%$ died. This differs from our earlier series ${ }^{12}$ of patients in whom admission GCS scores were 3 to 5: approximately one half of the surgically treated patients survived, but a good outcome was observed in only $10 \%$. As we have previously reported, although surgery was significantly associated with death, there was no relationship to morbidity in patients with admission GCS scores of 6 to 8. Patients with admission GCS scores of 9 to 11 fared the same after surgery, with $83 \%$ surviving and $46 \%$ making a good outcome. Nonetheless, statistical analysis revealed that surgery did not significantly affect outcome in the group of patients with GCS scores of 9 to 11 . Patients with admission GCS scores of 12 to 15 did well after surgery with 96\% surviving and $80 \%$ making a good outcome.

Because one in four surgically treated patients with GCS scores of 6 to 8 made a good outcome after surgery, one consideration would be to improve surgical and medical management protocols rather than to abandon the effort to help these individuals by surgical intervention. Surgery may be appropriate in these cases if there is no evidence of SAH or other variables that may be strongly associated with a poor outcome. The markedly higher rate of good outcomes in surgically treated group of patients with GCS scores of 6 to 8 (24\% compared with $0 \%$ in nonoperative patients) indicates that caution must be used when deciding which patients should not undergo aggressive treatment. In the five nonoperative patients with GCS scores of 6 to 8 there was a lower survival rate $(0 \%)$ than in the 127 nonoperative patients $(2 \%)$ with GCS scores of 3 to 5 we have previously reported on. ${ }^{12}$
An important goal in this area of research is to identify which patients will most benefit from intervention. The authors' experiences suggest that, with regard to functional outcome, operative intervention was not of clear benefit to patients with GCS scores of 6 to 8. Despite misgivings about not performing surgery in those patients with GCS scores of 6 to 8 , the author was unable to predict which patients would have made a good outcome after surgical management. Clearly there are more salvageable cases with GCS score of 6 to 8 than with GCS scores of 3 to 5. Although it is reasonable to make an effort to identify the salvageable cases in patients with GCS scores of 3 to 8 , it is likely that surgical efforts may have an impact only on those patients with admission GCS scores of 6 to 8 .

\section{CONCLUSIONS}

In an earlier study we reported that statistical analysis relating mortality rates to operative and nonoperative management in patients with admission GCS scores of 3 , 4 or 5 was remarkable for having determined a significant relationship between operative intervention and survival ( $\mathrm{p} \leq 0.000001$ ), although only two of the 23 survivors experienced good outcomes. ${ }^{12}$ In the present study we found a significant relationship between operative intervention and survival $(\mathrm{p} \leq 0.01)$ in patients with an admission GCS score of of 6 to 8 . No significant relationships between operative intervention and survival were found in patients with an admission GCS scores of 9 to 12 and 13 to 15 . We also found a significant relationship between operative intervention and morbidity ( $\mathrm{p} \leq 0.01)$ in patients with an admission GCS scores of 12 to 15 , whereas none was found in patients with an admission GCS score of 6 to 8 and 9 to 12 .

\section{References}

1. Aldrich EF, Eisenberg HM, Saydjari C, et al: Predictors of mortality in severely head-injured patients with civilian gunshot wounds: a report from the NIH Traumatic Coma Data Bank. Surg Neurol 38:418-423, 1992

2. Cavaliere R, Cavenago L, Siccardi D, et al: Gunshot wounds of the brain in civilians. Acta Neurochir 94:133-136, 1988

3. Clark WC, Muhlbauer MS, Watridge CB, et al: Analysis of 76 civilian craniocerebral gunshot wounds. J Neurosurg 65:9-14, 1986

4. Grahm TW, Williams FC Jr, Harrington T, et al: Civilian gunshot wounds to the head: a prospective study. Neurosurgery 27:696-700, 1990

5. Helling TS, McNabney WK, Whittaker CK, et al: The role of early surgical intervention in civilian gunshot wounds to the head. J Trauma 32:398-400, 1992

6. Kaufman HH, Levy ML, Stone JL, et al: Patients with Glasgow Coma Scale scores 3, 4, 5 after gunshot wounds to the brain. Neurosurg Clin North Am 6:701-714, 1995

7. Kaufman HH, Makela ME, Lee KF, et al: Gunshot wounds to the head: a perspective. Neurosurgery 18:689-695, 1986

8. Kaufman HH, Schwab K, Salazar AM: A national survey of neurosurgical care for penetrating head injury. Surg Neurol 36: 370-377, 1991

9. Kordestani RK, Counelis GJ, McBride DQ, et al: Cerebral arterial spasm after penetrating craniocerebral gunshot wounds: transcranial Doppler and cerebral blood flow findings. Neurosurgery 41:351-360, 1997 
10. Levi L, Linn S, Feinsod M: Penetrating craniocerebral injuries in civilians. Br J Neurosurg 5:241-247, 1991

11. Levy ML, Davis S: Outcome of aggressive surgical management in patients with low Glasgow Coma Scale scores following craniocerebral missile wounds, in Aarabi B, Kaufman HH, Dagi TF, et al (eds): Missile Wounds of the Head and Neck. Park Ridge, IL: American Association of Neurological Surgeons, 1999, Vol 1, pp 423-428

12. Levy ML, Masri LS, Lavine S, et al: Outcome prediction after penetrating craniocerebral injury in a civilian population: aggressive surgical management in patients with admission Glasgow Coma Scale scores of 3, 4, or 5. Neurosurgery 35:77-85, 1994

13. Levy ML, Rezai A, Masri LS, et al: The significance of subarachnoid hemorrhage after penetrating craniocerebral injury: correlations with angiography and outcome in a civilian population. Neurosurgery 32:532-540, 1993

14. Lillard PL: Five years experience with penetrating craniocerebral gunshot wounds. Surg Neurol 9:79-83, 1978

15. Martin NA, Doberstein C: Cerebral blood flow measurement in neurosurgical intensive care. Neurosurg Clin North Am 5: 607-618, 1994

16. Martin NA, Doberstein C, Alexander M, et al: Posttraumatic cerebral arterial spasm. J Neurotrauma 12:897-901, 1995

17. Martin NA, Doberstein C, Zane C, et al: Posttraumatic cerebral arterial spasm: transcranial Doppler ultrasound, cerebral blood flow, and angiographic findings. J Neurosurg 77:575-583, 1992

18. Nagib MG, Rockswold GL, Sherman RS, et al: Civilian gunshot wounds to the brain: prognosis and management. Neurosurgery 18:533-537, 1986
19. Polin RS, Shaffrey ME, Phillips CD, et al: Multivariate analysis and prediction of outcome following penetrating head injury. Neurosurg Clin North Am 6:689-699, 1995

20. Selden BS, Goodman JM, Cordell W, et al: Outcome of selfinflicted gunshot wounds of the brain. Ann Emerg Med 17: 247-253, 1988

21. Shaffrey ME, Polin RS, Phillips CD, et al: Classification of civilian craniocerebral gunshot wounds: a multivariate analysis predictive of mortality. J Neurotrauma 9 (Suppl 1): S279-S285, 1992

22. Sherman WD, Apuzzo MLJ, Heiden JS, et al: Gunshot wounds to the brain a civilian experience. West J Med 132:99-105, 1980

23. Siccardi D, Cavaliere R, Pau A, et al: Penetrating craniocerebral missile injuries in civilians: a retrospective analysis of 314 cases. Surg Neurol 35:455-460, 1991

24. Stone JL, Lichtor T, Fitzgerald LF: Gunshot wounds to the head in civilian practice. Neurosurgery 37:1104-1112, 1995

25. Suwanwela C, Suwanwela N: Intracranial arterial narrowing and spasm in acute head injury. J Neurosurg 36:314-323, 1972

26. Weir B, Grace M, Hansen J, et al: Time course of vasospasm in man. J Neurosurg 48:173-178, 1978

Manuscript received November 30, 1999.

Accepted in final form December 7, 1999.

Address reprint requests to: Michael L. Levy, M.D., Division of Pediatric Neurosurgery, University of Southern California School of Medicine, 1300 North Vermont Street, Suite 906, Los Angeles, California 90027. 